\title{
Design and Fabrication of a Responsive Carrier Component Envelope
}

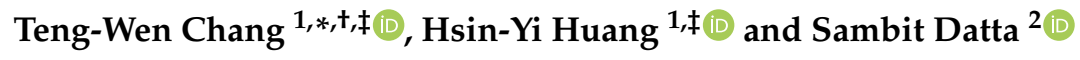 \\ 1 College of Design, National Yunlin University of Science and Technology, Taipei 10607, Taiwan; \\ sherry.hhy@gmail.com \\ 2 School of Electrical Engineering, Computing and Mathematical Sciences, Curtin University, \\ Bentley, WA 6102, Australia; Sambit.Datta@curtin.edu.au \\ * Correspondence: tengwen@yuntech.edu.tw; Tel.: +886-5-534-2601 (ext. 6510) \\ + Current address: 123 University Road, Section 3, Douliou, Yunlin 64002, Taiwan, R.O.C. \\ $\ddagger$ These authors contributed equally to this work.
}

Received: 28 February 2019; Accepted: 11 April 2019; Published: 15 April 2019

check for updates

\begin{abstract}
Responsive architecture comprises the creation of buildings or structural elements of buildings that adapt in response to external stimuli or internal conditions. The responsiveness of such structures rests on addressing constraints from multiple domains of expertise. The dynamic integration of geometric, structural, material and electronic subsystems requires innovative design methods and processes. This paper reports on the design and fabrication of a responsive carrier component envelope (RCCE) that responds by changing shape through kinetic motion. The design of the RCCE is based on geometry and structure of carrier surfaces populated with a kinetic structural component that responds to external stimuli. We extend earlier prototypes to design a modular, component-driven bottom-up system assembly exploring full-scale material and electronic subsystems for the expansion and retraction of a symmetric polar array based on the Hobermann sphere. We test the kinetic responsiveness of the RCCE with material constraints and simulate responses by connecting the adaptive components with programmable input and behavior. Finally, a concrete situation from practice is presented where 16 fully-functional components of the adaptive component are assembled and tested as part of an interactive public placemaking installation at the Shenzhen MakerFaire Exhibition. The RCCE experimental prototype provides new results on the design and construction of an adaptive assembly in system design and planning, choice of fabrication and assembly methods and incorporation of dynamic forms. This paper concludes that the design and assembly of an adaptive structural component based on RCCE presents results for designing sensitive, creative, adaptable and sustainable architecture.
\end{abstract}

Keywords: responsive architecture; kinetic envelope; adaptive design; interactive architecture; moveable facade components; carrier component structures; sensor interaction; digital fabrication

\section{Introduction}

Buildings or elements of buildings that offer adaptive features with the ability to adjust to changes in environmental conditions or external stimuli are termed responsive. Responsive envelopes act as an inter-media [1] between inside and outside, triggering changes that enhance the awareness and experience of place as well as performing functions such as modulation of thermal comfort or lighting. Responsive components can be defined as "all those elements of the building that adapt to the needs of people as well as changes in the environment" [2]. The most common embodiment of responsive envelopes are structures or architectural elements that adapt to changes in climatic variables. 
These components may be high tech systems that employ sensor networks and actuators to monitor the environment and automate control of operable building elements. The Tower of Winds [3] is a cylindrical urban installation, clad in perforated aluminium panels. The responsiveness of the cylindrical envelope is achieved at night by translating the varying sound and wind levels into light through computational methods and translating these sensor data into powering different light emitting devices. The variability of the environment is thus directly made visible in the architecture such that changes in wind speed and noise levels reflect changes in the cylindrical envelope. In this way, the Tower of Winds is constantly transforming, with its small lamps changing colors according the surrounding sounds and its neon rings rippling according to the winds of the city. A second line of development includes dynamic or kinetic structures that respond by reconfiguring their physical shape or form. Aegis hyposurface [4] is a dynamic mechanical wall surface that deforms in response to stimuli. The prototype of faceted metallic panels deform physically as a real time response to electronic stimuli from the environment (movement, sound, and light). Each component of the surface is driven by a bed of pneumatic pistons, generating real-time dynamic terrains.

The design and assembly of responsive structures requires new levels of integration across geometric, structural, material and electronic subsystems. This paper reports on the design and fabrication of a responsive carrier component envelope (RCCE). In this paper, we address the design and fabrication of responsive envelopes that responds to sensing people by changing shape through kinetic motion. The design of the RCCE is based on geometry and structure of carrier surfaces populated with a kinetic structural component that responds to external stimuli. We extend earlier prototypes to design a modular, component-driven bottom up Design-For-Assembly (DFA) system exploring full-scale integration of material, structural, kinetic and electronic subsystems. We present the expansion and retraction of the symmetric polar array based on the Hobermann sphere, test the kinetic responsiveness of the RCCE with material constraints and simulate responses by connecting the adaptive components with programmable sensor input and real-time dynamic behavior. Finally, we prototype and assemble an aggregation of fully functional components as part of an interactive public placemaking installation.

\subsection{Background}

With the evolution of information technology and smart materials, new categories of responsive intelligent skins have been proposed. Responsive envelopes have been broadly categorized into media facades [5], dynamic envelopes [6,7] and interactive systems [8,9]. The role of the architectural skin as a responsive element [10,11] is a central metaphor in both traditional responsive building facades [12] as well as emergent intelligent facade design [13].

Advances in flexible, configurable geometric representations [14-16] have been central to the design of responsive components. Flexible parametric models permit both design space exploration [17,18] and mapping of digital models to physical infrastructure [19]. Through inexpensive hardware components and embedded sensor systems, virtual simulations can be connected directly to physical models [20]. Sophisticated human-computer interaction paradigms such as mixed-initiative [21] dynamic interfaces [22] and distributed interaction [23] enable responsive components and their interaction with complex environmental changes as well as internal and external stimuli. Furthermore, three-dimensional printing, laser cutting, and desktop milling allow the digital fabrication of components [24,25] for rapid assembly and prototyping [26].

Sensors are commonly used to track indoor and outdoor environmental variables as well as recognize activity patterns and spatial distributions. Kinetic Architecture present concepts of responsiveness where secondary environmental systems can be attached to a primary structural system combined into a collective behavioral system [27]. The stochastic rotation of tiles [28] and the kinetic behavior of origami techniques with shape memory alloy actuators [29] and dynamic skins [30] have been developed. Structures that respond to lighting and energy optimization have 
been proposed [31-33]. Parametric models driven by algorithms for exploring kinetic facade design for daylighting performance [34], wind motion [35] and dynamic shading [36] have been reported.

\subsection{Motivation}

As described above, current models of responsive components lack the integration of variable geometry and do not include carrier component structures. To address these shortcomings, we propose the integration of geometric, structural and electronic subsystems supporting discrete adaptive motion within geometric constraints. Such a responsive component dynamically integrates environmental information in real time, and responds by changing its geometric state through structural integration, decomposable surface and open reprogrammable components. The design and assembly of a RCCE are based on the following features:

Support Geometry. The support grid and overall geometry should be defined by a carrier surface geometry [37]. The carrier surface is a self-supporting, integrated structural grid geometry, avoiding peripheral structural or cladding elements. The support system builds on our previous work on carrier component surfaces [38] and responsive carrier component envelopes [39].

Discretization. The carrier surface should be decomposable into an aggregation of discrete repeatable components. Self similar kinetic components are aggregated to minimize cost and complexity and provide a coherent integration between geometry/motion, responsive/electronic and physical/structural systems. The RCCE discretization builds on our previous work component aggregation including representative volume elements [38] and carrier component envelopes [28].

Open reprogrammable components. The carrier-component structure should support open ended reprogrammable sensor input-output for responsiveness to internal and external change parameters. Components should be reprogrammable and reconfigurable to allow a broader range of experiments with interactivity. The responsive methodology developed in $[39,40]$ is used to develop the responsive/electronic subsystem of sensor networks.

In the next section, we present the design and assembly of a RCCE made feasible through advances in the integration of geometric, material and electronic subsystems as described above.

\section{Materials and Methods}

This section describes the geometry and control aspects of the the RCCE experimental prototype covering the three features identified above, namely support geometry, incorporation of adaptive motion through discrete components and reprogrammable interaction.

The project was undertaken in collaboration between IdeaFactory (YunTech) and CodeLab (Curtin University) under the supervision of Carl Yu (OneWork.io), a startup company with expertise on Internet of Things (IoT). The Curtin team focused on the design of the carrier surface and kinetic component prototyping while The YunTech team focused on the fabrication and making of the RCCE and put together prototypes, improved aspects of geometry and code and took part in the final assembly. All three teams cooperated remotely on design and fabrication of the prototype, improved aspects of kinetic geometry, material development code and took part in the final assembly. To accomplish the task, three teams worked simultaneously in different locations, namely structure/carrier component design, fabrication process refinement and digital fabrication factory culminating in a three-day public installation assembly at the Shenzhen MakerFaire Exhibition. In the next sections, we describe the design and fabrication of the RCCE.

\subsection{Carrier Component Geometry}

The design geometry of the RCCE was developed with both top-down and bottom-up approaches using modeling and scripting techniques. The structural grid fabrication was based on the approach presented by Andres Sevtsuk and Raul Kavlo in the SUTD pavilion [41]. The design is a modular, component-driven bottom-up system assembly exploring full-scale material and electronic subsystems. 
The top-down approach involved creation of a grid shell surface structure for carrier surface. The outcomes of the carrier component design process are summarized in Figure 1.

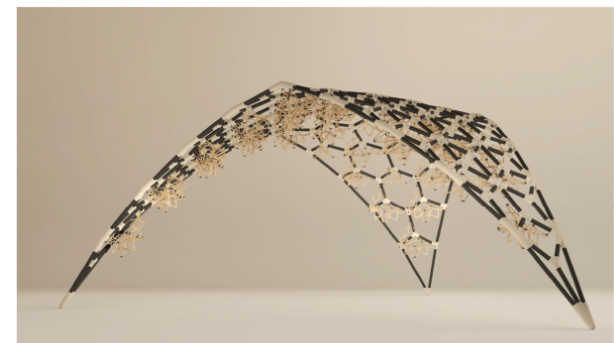

(a)

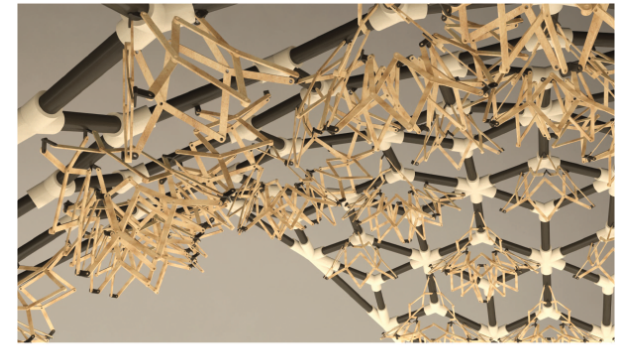

(b)

Figure 1. Carrier component structure: (a) detailed design of a discrete carrier surface as a support structure; and (b) aggregation of a single adaptive component over the surface.

This presents significant challenges in terms of assembly of complex interactive components. One of the challenges to the introduction of a curved structural grid was variation in the geometry of parts. In the bottom-up approach, the component scissor truss assembly was derived from four sides of a dodecahedron in Grasshopper. The movement of the arms of the scissors was simulated using a spring system in Kangaroo (Figure 2).

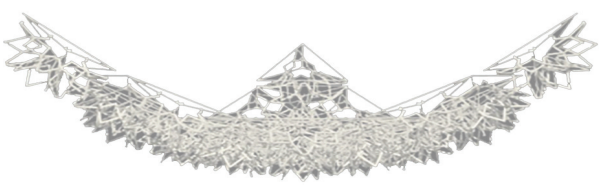

(a)

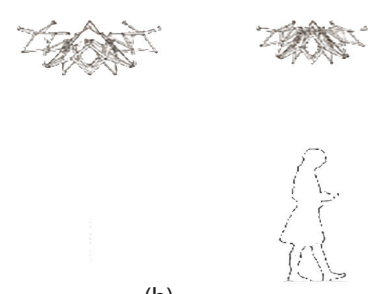

(b)

Figure 2. (a) Design rendition of responsive envelope on carrier surface; and (b) adaptive component triggering closed and open states through motion.

\subsection{Adaptive Component}

The components followed rules of local independence and global correspondence to form iterative responses and spatial configurations based on various local inputs. The moveable components edre based on the Hoberman Sphere and provided an adaptive assembly constrained to open and close in response to sensing change in the environment. The next step was to standardize kinetic component parts, namely the guiding rods to fit the curving surface. The self-organization was constrained by surface geometry on which the component was aggregated. Expansion and retraction of a symmetric polar array based on the Hobermann sphere was investigated. Parts had a degree of freedom in a complete system and worked independently while responding to their neighbors effecting changes on the larger scales, as simulated in Figure 3.

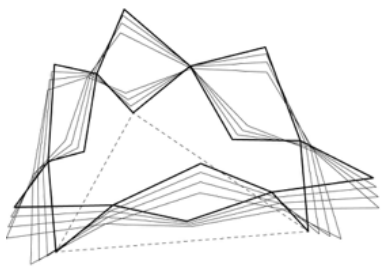

ico edge: $320-240 \mathrm{~mm}$

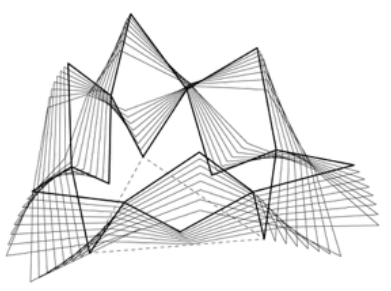

ico edge: $320-160 \mathrm{~mm}$

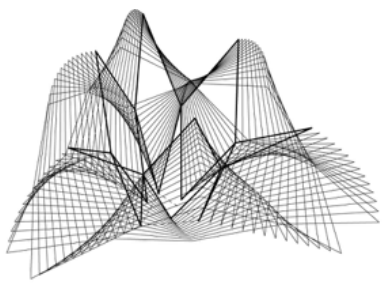

ico edge: $320-40 \mathrm{~mm}$

Figure 3. The design development of the adaptive component simulation of the scissor truss motion using a spring system simulation. 
We tested the kinetic responsiveness of the RCCE with material constraints and simulated responses by connecting the adaptive components with programmable input and behavior. This allowed for less deterministic part-to-whole relationships to emerge from interactions of local protocols and a multitude of external stimuli. In the current prototype, components in RCCE are comprised of scissor truss assembly based on Hoberman sphere guided by sliding arms and allow a single degree of freedom in a symmetrically polar array, as shown in Figure 4.
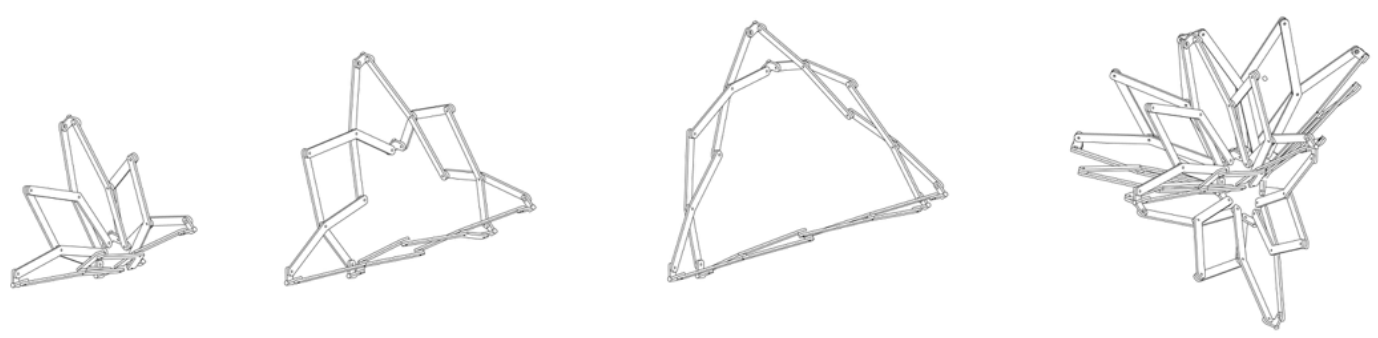

Figure 4. Detailed design modeling of polar array assemblies based on the expansion and contraction principles of a Hobermann sphere.

A standardization script was developed that fitted equilateral triangles using geometric transformation per face. Each component was comprised of a scissor truss assembly based on Hoberman sphere guided by sliding arms and allowed a single degree of freedom in a symmetrically polar array. The complexity of the assembly and its kinetic actuation, variation of parameters and scale were tested through material fabrication, as shown in Figure 5.
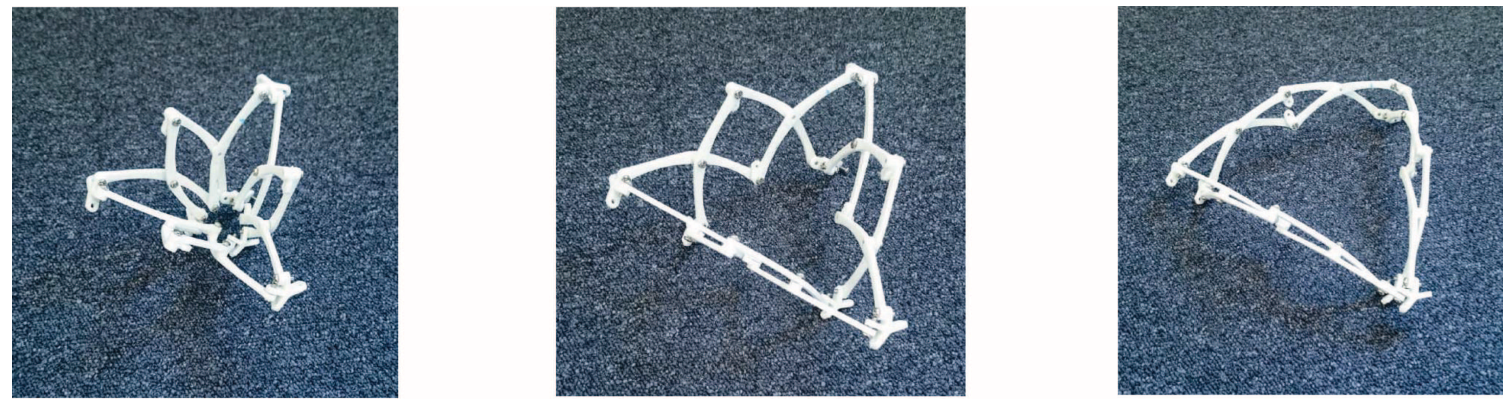

Figure 5. Physical prototype models with variational play to understand the stages of motion from closed to open.

The dynamic component was based on a planar surface tessellation with equilaterals and separated from the structural support grid. The adaptive component configuration prototype model was based on the scissor truss motion of a Hobermann sphere. This design compromise simplified the kinetic component and enabled the delivery of local interactions without structural ramifications. The self-organization was constrained by surface geometry, on which the component was aggregated. Parts had a degree of freedom in a complete system and worked independently while responding to the neighbors effecting changes on the larger scales. This allowed for a less deterministic part-to-whole relationship to emerge from interactions of local protocols and a multitude of external stimuli (Figure 6).

A few 3D-printed components were used to connect assembly at key intersections with unique angles. In this case, 3D printing was used as part of the combinatorial approach to construction where many different materials and parts came together as oppose to creating complex, continuous forms (Figure 7). 


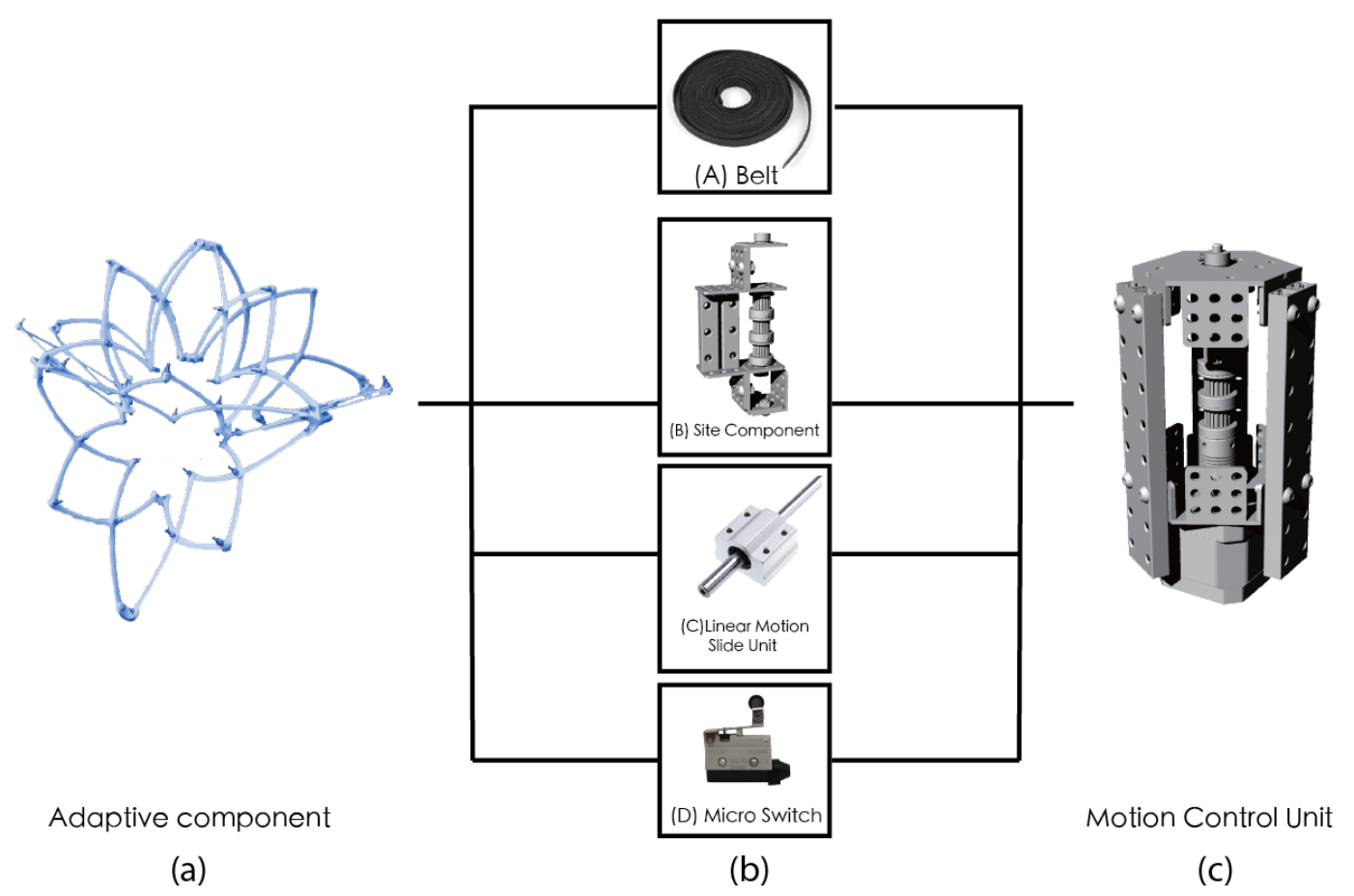

Figure 6. (a) Adaptive component configuration prototype model based on the scissor truss; (b) MakeBlock components for kinetic motion; and (c) central motion control unit.

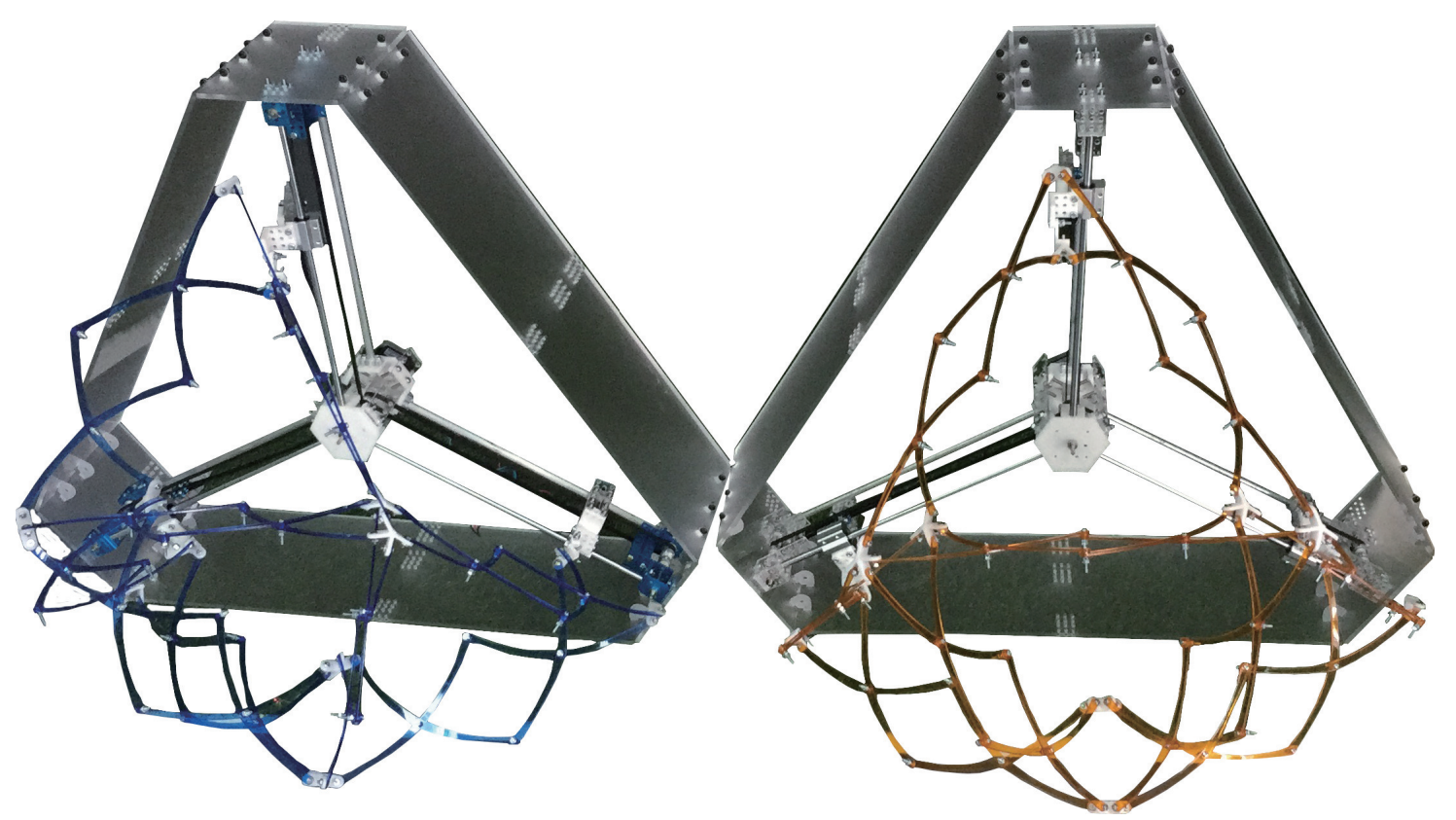

Figure 7. Early component configuration prototype models for kinetic components based on the scissor truss.

Early prototypes contained springs that kept component in tension while the final prototype used timing belts and motors to control position more precisely and eliminated kinetic energy, which made motion more unstable given relatively large number of parts per truss. The design used a number of standard industrial components such as steel shafts and connector brackets to reduce construction time and cost (Figure 7). 


\subsection{Kinetic Component Fabrication}

The basic unit was built on a triangle containing tetrahedral scissor truss geometry (Figure 8). Structurally, the components were made of three main parts: (1) the structural frame that housed all the parts and connects it to its neighbors; (2) a guiding rail assembly that contained all sensing, actuating and logic components; and (3) kinetic scissor truss assembly. The 34:1 motor connected to three linear belts that guided the base points of the truss. There was a binary switch that communicated to the script terminal position of the truss under maximum contraction. A flexible multi-axis 3D-printed connector allowed us to connect the kinetic scissor truss to a planar rail frame that could be animated by linear motion.

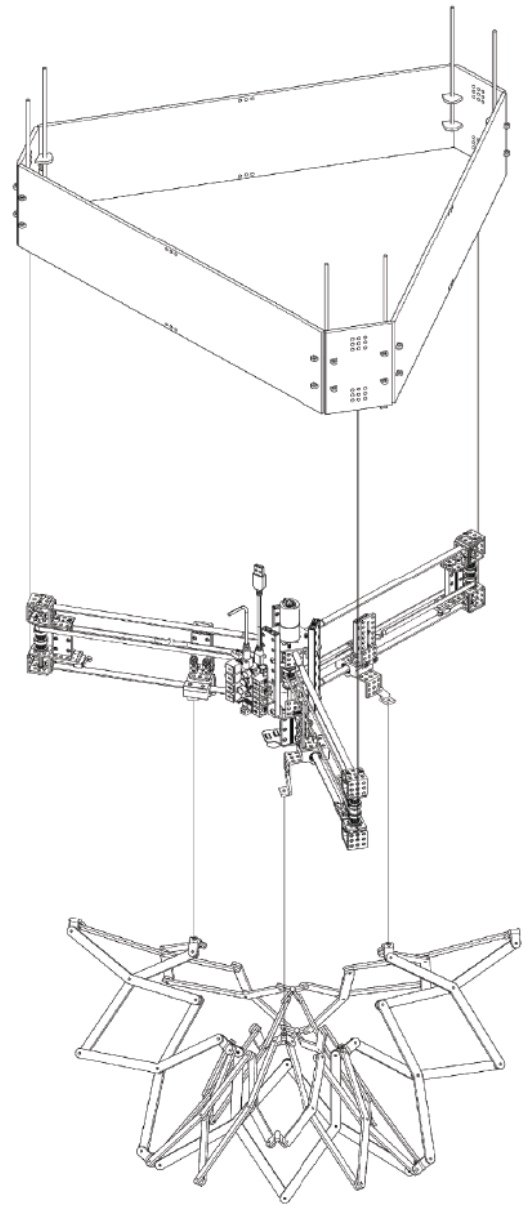

(a)

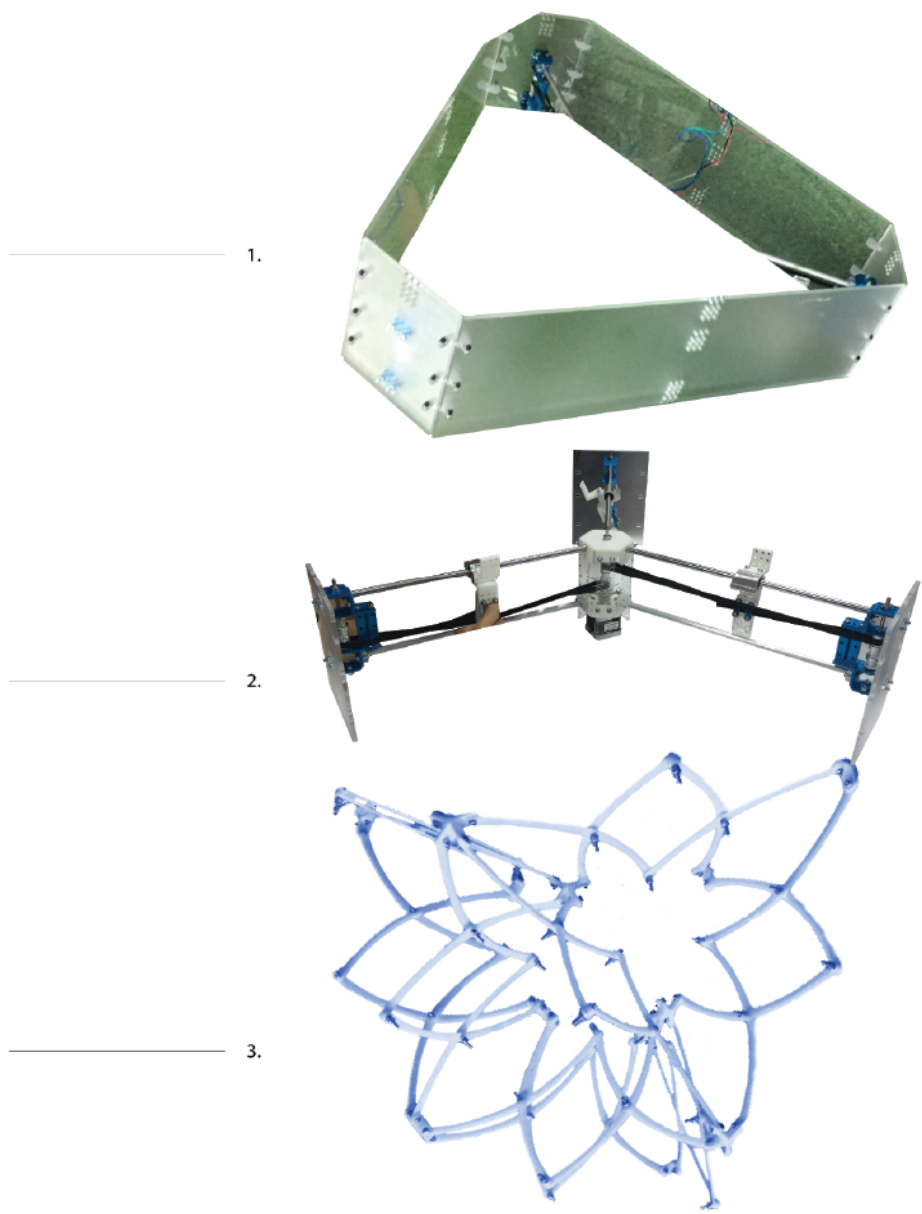

(b)

Figure 8. (a) RCCE design was done in three subcomponents comprising connectors, motion bars and scissor truss configuration; and (b) view of the final adaptive component assembly and fabrication.

An ultrasonic sensor (HC-SR04) driven by Arduino microcontroller detected proximity of people in front of it with a maximum range of about $5 \mathrm{~m}$. Upon detection, it activates a geared motor connected to the kinetic truss through linear motion and began contracting it. Once the sensor stopped detecting or the collision switch on one of the arms was hit with the truss arm, the script pauses and proceeded to return the mechanism to its extended configuration. The control system used standard Arduino IDE written in Cal frame. The microcontroller was connected to the motor, ultrasonic sensor, an LED strip and a switch to determine position of the kinetic truss. This allowed components to "sense" presence and proximity and respond by retraction or contraction. The precise timing allowed for real-time control and synchronization of motor positions in relation to external stimuli. 


\subsection{Prototyping and Assembly}

The prototyping process used MakeBlock [42] components for standard mechanical parts and electronic modules such as sensors, control boards, motor drivers and communicators. The fabrication process was undertaken experimentally using a trial-and-error process, with the fabrication team iteratively testing all control mechanism and parts. The laser cut process took the 3D design to create 2D sheet layouts via Grasshopper scripts. The non-standard parts were laser-cut Plexiglas elements for peripheral structural arms and 3D-printed components for kinetic mechanism and connectors not available from the MakeBlock component library. The 3D printer for the kinetic mechanism components used two materials, PLA and wax, for different parts due to different requirements for assembly. For instance, the gear for timely belt control required more accurate model, thus we used wax 3D print, but it is softer. The central part of gear for motor to spin needed a tighter connection for headless setscrew and metal shaft pin, thus we decided to use PLA.

A few 3D-printed components were used to connect assembly at key intersections with unique angles. In this case, 3D printing was used as part of the combinatorial approach to construction where many different materials and parts came together as opposed to creating complex, continuous forms (Figure 9).

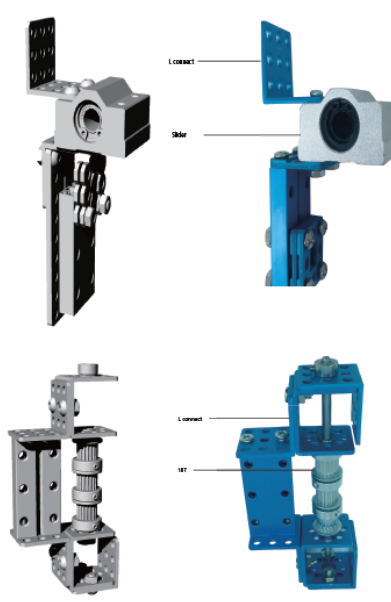

(a)

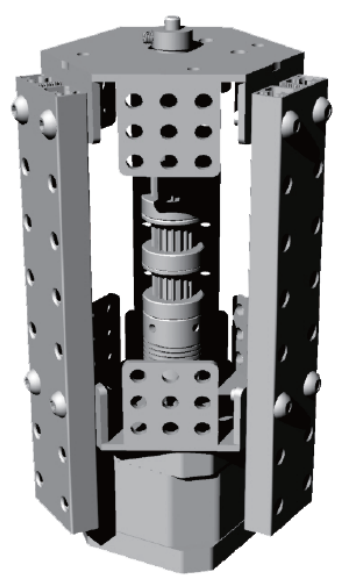

(b)

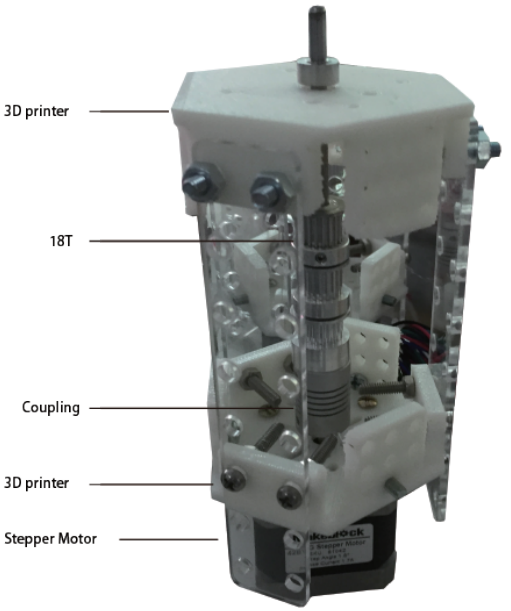

(c)

Figure 9. (a) Three subcomponents comprising connectors, motion bars and scissor truss configuration; (b) RCCE motion control unit; and (c) component assembly and fabrication.

Over the period of three weeks, the teams communicated and exchanged 3D models, scripts and other documentation relating to prototype construction. The Curtin team assembled design documentation, while Yun Tech team prototyped various design iterations using digital fabrication equipment at their facilities.The fabrication process took places in IDF at Yunlin, Taiwan and test assembly process in factory first and then all 16 sets were assembled on site in Shenzhen. Many factors affected the prototyping and assembly process:

- Tolerance and accuracy of parts via fabrication machine

- Mechanical control of movement via electronic boards

- Mechanical movement and vibration

- Parts assembly direction, thickness of material and its durability

- Friction between different parts 
These factors affected the assembly and fabrication process and required the testing of multiple fabrication and assembly methods iteratively in a very short period time. The design team also needed to change the original design to accommodate the difficulties of fabrication (Figure 10). An agile design process methodology provided transparent workflow to the entire team to understand the status of different activities as the project progressed. It also held team members accountable for what they were assigned and documented feedback directly from all remote teams.

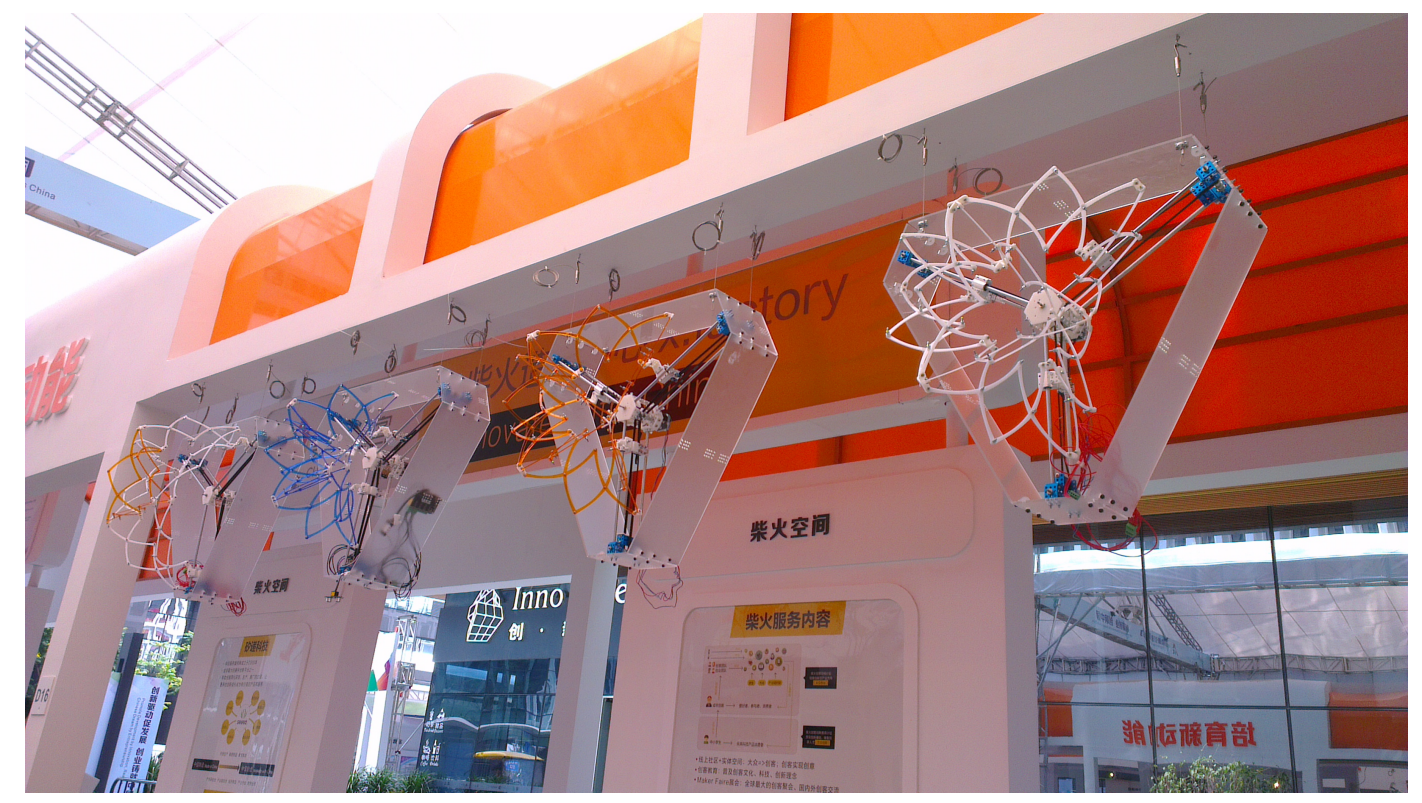

Figure 10. RCCE component prototype variations.

\subsection{Exhibition}

Finally, a concrete situation from practice was presented, where 16 fully functional components of the adaptive component were assembled and tested as part of an interactive public placemaking installation at the Shenzhen MakerFaire Exhibition. The aim of the RCCE was to develop an interactive media between people and space, as described in [1]. The RCCE in this sense represented an interactive media that received/sensed the interactive behaviors of people and reacted back into space. The behavior of people and the performative aspects of responsive installations were collected as video recordings on site and are provided in the Supplementary Materials to reflect the interaction between people and space. Design and research were conducted on multiple aspects of the process, remotely communicating between the teams in Shenzhen, Taiwan and Perth, Australia. The three teams worked simultaneously in different locations to develop structure/carrier component design, fabrication process refinement and digital fabrication, respectively. The full scale RCCE prototype was assembled in Shenzhen culminating in a three-day public installation assembly at the Shenzhen MakerFaire Exhibition.

Each component of the RCCE installation was an autonomous mechanism with an ultrasonic sensor (HC-SR04) driven by Arduino microcontroller. The sensor detected the proximity of objects (in this case people) in front of it with a maximum range of about $5 \mathrm{~m}$. Upon detection, it activated a geared motor connected to the kinetic truss through linear motion and began contracting it. Once the sensor stopped detecting or the collision switch on one of the arms was hit with the truss arm, the script paused and proceeded to return the mechanism to its extended configuration (Figure 11). 


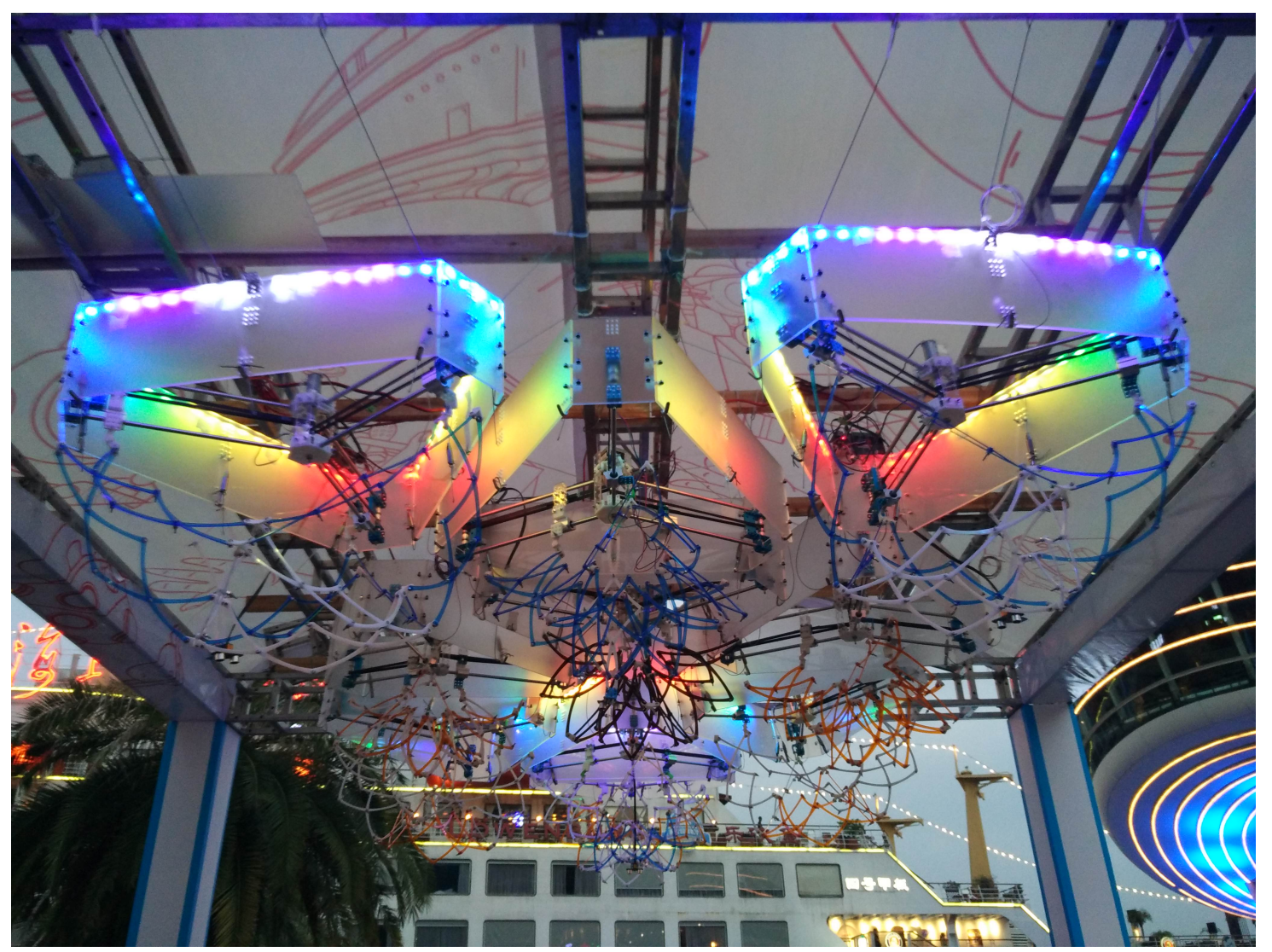

Figure 11. Full-scale RCCE prototype: Installation of RCCE on site at Shenzhen MakerFaire Exhibition.

\section{Results}

The experimental prototype installation provided new results into the design and construction of an adaptive RCCE assembly comprising repeatable components that operate on computation protocols and exhibit responsive kinesis. The research addressed key issues underlying RCCE prototypes using a design and fabrication process integrated with technical subsystems. The findings of the study cover system design and planning, choice of fabrication and assembly methods and incorporation of dynamic forms. First, the outcomes of our research propose that carrier component structures are a useful abstraction for the development of responsive architecture. They permit the design and aggregation of repeatable components as well as provide a metaphor for allowing discrete components to respond either as a single element or in combination. Second, detailed design, fabrication and assembly of a single adaptive component is a critical level of abstraction for addressing issues of structure, material complexity, analysis of motion and electronic control and sensing.

Finally, the public installation of an RCCE prototype at the Shenzhen MakerFaire Exhibition demonstrated the potential role of responsive architecture in the public domain. During the three-day exhibition, the installation performed without failure and thousands of citizens interacted with the kinetic motion. These activities were recorded using video and samples are provided in the Supplementary Materials. These outcomes are summarized in Figure 12. The results of the experiment for the development of a responsive skin prototype are as follows.

Multi-scalar structure. A multi-scalar approach is needed for the development of the carrier surface structure and its subsequent articulation into discrete components requiring geometric resolution at two scales. The tessellation of the carrier surface must be rationalized to account for both part-to-whole relationships as well as control of component variation and scaling. In the RCCE, we separated the structural grid from the component aggregation to achieve this. 
Digital to Physical translation. The selection of materials and their tectonic properties for components and connections is critical for responsive architecture. The digital-to-physical translation from rationalized geometry to material requires a consideration of their assembly.

Sensor Design. Sensor density, an important element in interaction design, is the resolution-density trade-off in the type of sensor components. Electronic components and sensors require careful consideration of the interaction logic, input-output behaviors and sizing and resolution of components. Denser sensor arrays produce greater resolution but create more complexity in terms of set up and fabrication as well as driving up costs.

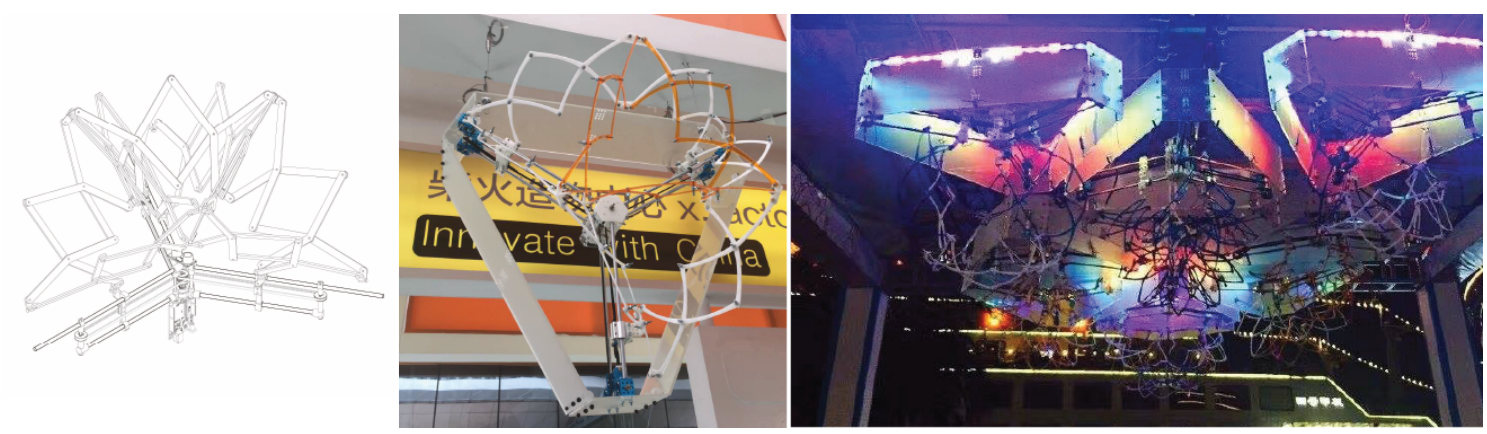

Figure 12. The outcomes of our research: (left) design research into the carrier component structures; (middle) detailed design, fabrication and assembly of a single adaptive component; and (right) public installation of an RCCE prototype at the Shenzhen MakerFaire Exhibition.

The protocols developed in the RCCE highlight the opportunities and consequences of how local adaptive components relate to the whole carrier envelope with multiple constraints and scale considerations.

\section{Discussion}

Responsive components are not a new phenomenon in architecture. Responsive architecture can be understood as any building or building component designed for adaptation to change. The RCCE is an experiment in responsive architectural design that gives us insight into construction of complex architectural assemblies incorporating structural, material, kinetic and electronic subsystems. RCCE is a work in progress and, due to its complexity and wide range of interdisciplinary knowledge required, will require expanded collaboration in the future.

The main problems encountered are structural and control issues, and the need to script and iteratively test a largely bottom-up system. Although the behavior is emergent, the form remains static. This problem will be addressed in further design iterations to control the local parameters influencing the surface tessellation and have more control over aggregation of local geometry into a larger whole. This could be expanded upon by looking at the way components connect, degrees of freedom allowed per component and the way cladding layer can be arranged more continuously rather than as a collection of disparate kinetic scissor truss assemblies. The future implementation will include communication protocol between components to allow for coordinated transformation of the structure. This tradition of design suggests that new ways of thinking about responsive components have their roots in a series of precedents that respond to change, such as the light sensitive apertures in the Institut du Monde Arabe in Paris, and they have the capacity to transform the way that architecture is experienced.

In conclusion, the design and assembly of adaptive components present new insights into designing sensitive, creative, adaptable, and sustainable architecture. Negroponte proposed that advances in artificial intelligence and the miniaturization of components would result in buildings capable of intelligently recognizing the activities of their users and responding to their needs, as well as changes in the external and internal environment [9]. There is a continuity in the design of automated building components, such as presented in this paper, and the long tradition of design in manually 
operated responsive mechanisms and static architectural elements that modulate inside-outside conditions. Meagher's study of the Maison de Verre shows how mechanical adjustment of components can achieve such a poetic responsiveness [2].

Supplementary Materials: The following are available online at http://www.mdpi.com/2075-5309/9/4/84/s1.

Author Contributions: Conceptualization, T.-W.C. and S.D.; methodology, T.-W.C.; software development, S.D.; validation, T.-W.C. and H.-Y.H.; formal analysis, all authors; writing-original draft preparation, S.D.; writing-review and editing, T.-W.C.; visualization, H.-Y.H.; supervision, T.-W.C.; project administration, H.-Y.H.; and funding acquisition, T.-W.C.

Funding: This research was funded by Idea Factory, YunTech, Taiwan and Curtin University, Australia.

Acknowledgments: The project was undertaken in collaboration between Idea Factory (Yunlin, Taiwan) and Code Lab (Perth, Australia) under the supervision of OneWork.io (Taipei, Taiwan), a startup company with expertise on Internet of Things (IoT).The authors acknowledge the contributions of the team leader of the project "Dynamic Cloud" Huei-Sheng Yu (onework.io) and the following participants: Andrei Smolik and Rex Auyeung (Curtin University); Tsai-Ling Hsieh, Yeong-Shenn Lee, Yun-Ru Chen and Chun-Yen Chen (Idea Factory, YunTech); Wayne Lin and Monica Shen (x.factory).

Conflicts of Interest: The authors declare no conflict of interest.

\section{References}

1. Bloomer, K.C.; Moore, C.W.; Yudell, R.J.; Yudell, B. Body, Memory, and Architecture; Yale University Press: New Haven, CT, USA, 1977.

2. Meagher, M. Designing for change: The poetic potential of responsive architecture. Front. Arch. Res. 2015, 4, 159-165. [CrossRef]

3. Ito, T. Blurring Architecture, 1971-2005: Rethinking the Relationship between Architecture and the Media; Art Books International London Milan: Charta, Colombia, 1999.

4. Goulthorpe, M.; Burry, M.; Dunlop, G. Aegis Hyposurface $(\subset):$ The Bordering of University and Practice. In Proceedings of the Twenty First Annual Conference of the Association for Computer-Aided Design in Architecture, ACADIA, CUMINCAD, New York, NY, USA, 11-14 October 2001; pp. 334-349.

5. Mignonneau, L.; Sommerer, C. Media Facades as Architectural Interfaces. In The Art and Science of Interface and Interaction Design; Sommerer, C., Jain, L.C., Mignonneau, L., Eds.; Studies in Computational Intelligence; Springer: Berlin/Heidelberg, Germany, 2008; pp. 93-104, doi:10.1007/978-3-540-79870-5_6.

6. Schittich, C. Building Skins: Concepts, Layers, Materials; Edition Detail; Birkhäuser: Munich, Germany, 2001.

7. Wigginton, M.; Harris, J. Intelligent Skins; Routledge: Abingdon, UK, 2013.

8. Tomitsch, M.; Grechenig, T.; Moere, A.V.; Renan, S. Information Sky: Exploring the Visualization of Information on Architectural Ceilings. In Proceedings of the 2008 12th International Conference Information Visualisation, London, UK, 9-11 July 2008; pp. 100-105, doi:10.1109/IV.2008.81. [CrossRef]

9. Sterk, T.d. Building upon Negroponte: A hybridized model of control suitable for responsive architecture. Autom. Constr. 2005, 14, 225-232, doi:10.1016/j.autcon.2004.07.003. [CrossRef]

10. Desai, A. Designing Building Skins. Master's Thesis, Dept. of Architecture, Massachusetts Institute of Technology, Cambridge, MA, USA, 1992.

11. Srisuwan, T. Fabric Façade: An Intelligent Skin. Int. J. Build. Urban Inter. Landsc. Technol. BUILT 2017, 9, 7-13.

12. Koudlai, A. In and Out of Control: An Exercise in the Design of LOW-tech/High Effect Responsive Building Facades; State University of New York at Buffalo: Buffalo, NY, USA, 2016.

13. Capeluto, G.; Ochoa, C.E. Intelligent Envelopes for High-Performance Buildings: Design and Strategy; Springer: Berlin, Germany, 2016.

14. Pottmann, H. Architectural Geometry; Bentley Institute Press: Exton, PA, USA, 2007.

15. Hudson, R. Parametric Development of Problem Descriptions. Int. J. Arch. Comput. 2009, 7, 199-216. [CrossRef]

16. Woodbury, R. Elements of Parametric Design; Routledge: London, UK; New York, NY, USA, 2010.

17. Woodbury, R.; Burrow, A.; Datta, S.; Chang, T.W. Typed feature structures and design space exploration. Artif. Intell. Eng. Des. Anal. Manuf. 1999, 13, 287-302. [CrossRef] 
18. Aish, R.; Woodbury, R. Multi-level Interaction in Parametric Design. In Smart Graphics; Butz, A., Fisher, B., Krüger, A., Olivier, P., Eds.; Number 3638 in Lecture Notes in Computer Science; Springer: Berlin/Heidelberg, Germany, 2005; pp. 151-162.

19. Jeng, T., Designing a ubiquitous smart space of the future: The principle of mapping. In Design Computing and Cognition'04; Springer: Berlin, Germany, 2004; pp. 579-592.

20. Kim, D.Y.; Kim, S.A. An exploratory model on the usability of a prototyping-process for designing of Smart Building Envelopes. Autom. Constr. 2017, 81, 389-400. [CrossRef]

21. Datta, S. Modeling dialogue with mixed initiative in design space exploration. Artif. Intell. Eng. Des. Anal. Manuf. 2006, 20, 129-142, doi:10.1017/S0890060406060124. [CrossRef]

22. Jiang, H.; Chang, T.W.; Liu, C.L. Musical Skin: A Dynamic Interface for Musical Performance. In Human-Computer Interaction. Interaction Techniques and Environments; Jacko, J., Ed.; Lecture Notes in Computer Science; Springer: Berlin/Heidelberg, Germany, 2011; Volume 6762, pp. 53-61, doi:10.1007/978-3-642-21605-3_6.

23. Chang, T.W.; Datta, S.; Lai, I.C. Modelling Distributed Interaction with Dynamic Agent Role Interplay System. Int. J. Digit. Media Des. 2016, 8, 1-14.

24. Seely, J.C. Digital Fabrication in the Architectural Design Process. Ph.D. Thesis, MIT, Cambridge, MA, USA, 2004.

25. Kolarevic, B. Back to the Future: Performative Architecture. Int. J. Arch. Comput. 2004, 2, 43-50. [CrossRef]

26. Huang, H.Y.; Chang, T.W.; Wu, Y.S.; Chen, J.Y. Collective Fabrication A Responsive Dynamic Skin Design Case. In Proceedings of the 22nd International Conference on Computer Aided Architectural Design Research in Asia; Janssen, P., Loh, P., Raonic, A., Schnabel, M.A., Eds.; CAADRIA: Singapore, 2017; pp. 99-100.

27. Foged, I.W.; Kirkegaard, P.H.; Christensen, J.T.; Jensen, M.B.; Poulsen, E.S. Shape Control of Responsive Building Envelopes. In Proceedings of the International Symposium of the International Association for Shell and Spatial Structures (IASS): Spatial Structures-Temporary and permanent, Shanghai, China, 8-12 November 2010; IASS, China Architecture and Building Press: Beijing, China, 2010; pp. 2602-2609.

28. Datta, S.; Hanafin, S.; Woodbury, R.F. Responsive envelope tessellation and stochastic rotation of 4-fold penttiles. Front. Arch. Res. 2014, 3, 192-198, doi:10.1016/j.foar.2014.03.002. [CrossRef]

29. Pesenti, M.; Masera, G.; Fiorito, F.; Sauchelli, M. Kinetic solar skin: A responsive folding technique. Energy Procedia 2015, 70, 661-672

30. Chang, T.W.; Jiang, H.; Chen, S.H.; Datta, S. Dynamic Skin: Interacting with Space. In Proceedings of the 17th International Conference on Computer Aided Architectural Design Research in Asia, CAADRIA, Chennai, India, 25-28 April 2012; pp. 89-98.

31. Chen, X. Interactive Pavillions: Responsive Transformation of Structure Systems. Thesis Prep, School of Architecture, Syracuse University, Syracuse, NY, USA, 2015.

32. Shaviv, E. Integrating energy consciousness in the design process. Autom. Constr. 1999, 8, 463-472, doi:10.1016/S0926-5805(98)00101-0. [CrossRef]

33. Radford, A.D.; Gero, J.S. Design by Optimization in Architecture, Building, and Construction; Van Nostrand Reinhold: New York, NY, USA, 1988.

34. El Sheikh, M.M. Intelligent Building Skins: Parametric-Based Algorithm for Kinetic Facades Design and Daylighting Performance Integration; University of Southern California: Los Angeles, CA, USA, 2011.

35. Datta, S.; Hanafin, S.; Pitts, G. Experiments with stochastic processes: Facade subdivision based on wind motion. Int. J. Arch. Comput. 2009, 7, 389-402. [CrossRef]

36. Chang, T.W.; Datta, S. Dynamic Envelopes: Structural and Interactive transformations. In Proceedings of the 7th CUTSE Conference, Sarawak, Malaysia, 1 August 2012; pp. 89-98.

37. Pitts, G.; Datta, S. Parametric Modelling of Architectural Surfaces. In Proceedings of the 14th International Conference on Computer Aided Architectural Design Research in Asia, Yunlin, Taiwan, 22-25 April 2009; Chang, T.W., Champion, E., Chien, S.F., Chiou, S.C., Eds.; CAADRIA: Yunlin, Taiwan, 2009; pp. 635-644.

38. Hanafin, S.; Pitts, G.; Datta, S. Non-Deterministic Exploration through Parametric Design. Int. J. Arch. Comput. 2009, 7, 605-622. [CrossRef]

39. Smolik, A.; Chang, T.W.; Datta, S. Prototyping Responsive Carrier-Component Envelopes. In Proceedings of the 22nd CAADRIA Conference, Suzhou, China, 5-8 April 2017; Janssen, P., Loh, P., Raonic, A., Schnabel, M.A., Eds.; CAADRIA: Suzhou, China, 2017; pp. 521-528. 
40. Datta, S.; Andrei, S.; Chang, T.W. Responsive Interaction in Dynamic Envelopes with Mesh Tessellation. In CAADence in Architecture International Workshop and Conference; Szoboszlai, M., Ed.; Dept. of Architecture and Interior Architecture: Budapest, Hungary, 2016; pp. 241-246.

41. Sevtsuk, A.; Kalvo, R. A freeform surface fabrication method with 2D cutting. Simul. Ser. 2014, 46, 138-145.

42. Makeblock Co., Ltd. Makeblock. Available online: http://learn.makeblock.com/en/ (accessed on 12 October 2016).

(C) 2019 by the authors. Licensee MDPI, Basel, Switzerland. This article is an open access article distributed under the terms and conditions of the Creative Commons Attribution (CC BY) license (http:/ / creativecommons.org/licenses/by/4.0/). 